\title{
Transcriptional Targeting of Replication-defective Adenovirus Transgene Expression to Smooth Muscle Cells In Vivo
}

\author{
Steven Kim, ${ }^{\star \ddagger}$ Hua Lin, ${ }^{\star}$ Eliav Barr, ${ }^{*}$ Lien Chu, ${ }^{\star}$ Jeffrey M. Leiden, ${ }^{\star \ddagger}$ and Michael S. Parmacek ${ }^{\star}$ \\ $*$ Department of Medicine and ${ }^{\ddagger}$ Department of Pathology, The University of Chicago, Chicago, Illinois 60637
}

\begin{abstract}
Gene transfer using replication-defective adenoviruses (RDAd) holds promise for the treatment of vascular proliferative disorders, but is potentially limited by the capacity of these viruses to infect multiple cell lineages. We have generated an RDAd vector, designated AdSM22-lacZ, which encodes the bacterial $l a c Z$ reporter gene under the transcriptional control of the smooth muscle cell (SMC)-specific SM22 $\alpha$ promoter. Here, we show that in vitro AdSM22-lacZ programs expression of the lac $Z$ reporter gene in primary rat aortic SMCs and immortalized A7r5 SMCs, but not in primary human umbilical vein endothelial cells (HUVECs) or NIH 3 T3 cells. Consistent with these results, after intraarterial administration of AdSM22-lacZ to control and balloon-injured rat carotid arteries, $\beta$-galactosidase activity was detected within SMCs of the tunica media and neointima, but not within endothelial or adventitial cells. Moreover, intravenous administration of AdSM22-lacZ did not result in lac $Z$ gene expression in the liver or lungs. Finally, we have shown that direct injection of AdSM22-lacZ into SMC-containing tissues such as the ureter and bladder results in high-level transgene expression in visceral SMCs. Taken together, these results demonstrate that transgene expression after infection with an RDAd vector can be regulated in an SMC lineage-restricted fashion by using a transcriptional cassette containing the SMC-specific SM22 $\alpha$ promoter. The demonstration of an efficient gene delivery system targeted specifically to SMCs provides a novel means to restrict expression of recombinant gene products to vascular or visceral SMCs in vivo. (J. Clin. Invest. 1997. 100:1006-1014.) Key words: smooth muscle • adenovirus • gene therapy • atherosclerosis • vascular diseases
\end{abstract}

\section{Introduction}

The capacity of smooth muscle cells $(\mathrm{SMCs})^{1}$ to proliferate and modulate their phenotype has been implicated in the pathogenesis of a number of diseases including atherosclerosis, restenosis after percutaneous transluminal coronary angioplasty, and asthma (for reviews see references 1-4). Gene transfer into SMCs holds promise both for elucidating the

Address correspondence to Michael S. Parmacek, M.D., University of Chicago, Room G611, MC 6088, 5841 S. Maryland Ave., Chicago, IL 60637. Phone: 773-702-2679; FAX: 773-702-2681; E-mail: parmacek@ medicine.bsd.uchicago.edu

Received for publication 11 April 1997 and accepted in revised form 10 June 1997.

J. Clin. Invest.

(c) The American Society for Clinical Investigation, Inc. 0021-9738/97/09/1006/09 \$2.00

Volume 100, Number 5, September 1997, 1006-1014

http://www.jci.org pathogenesis of these diseases and for their treatment (5-12). Replication-defective adenovirus vectors (RDAd) are particularly well suited for facilitating efficient gene transfer into SMCs in vivo. These viruses can be prepared at high titers and have been shown to efficiently infect SMCs both in vitro and in vivo $(13,14)$. In addition, RDAd display a relatively favorable safety profile. They do not integrate into the host genome and have not been associated with persistent infections or malignancies in humans (for review see reference 15). Several investigators have taken advantage of these properties to demonstrate that catheter-mediated delivery of RDAd encoding both cytotoxic and cytostatic proteins can be used to decrease SMC proliferation and neointima formation in both small and large animal models of vascular injury and restenosis $(5-7,12,16,17)$.

Although RDAd efficiently transduce both resting and proliferating SMCs in vivo, a potential limitation of their use in the clinical setting is their capacity to infect and program transgene expression in many different cell lineages and tissues (5, 15). For example, localized arterial administration of RDAd results in efficient infection of endothelial cells, vascular SMCs, and adventitial cells $(13,14)$. Moreover, intravenous administration of these vectors results in high-level gene transfer to the liver and lung (18-20). Several approaches have been used in an attempt to circumvent this problem. First, it has been possible to restrict the expression of a viral transgene to a specific cell or tissue by administering the virus ex vivo. However, this approach is laborious and is not practical for the treatment of most vascular proliferative disorders. A second approach has involved delivery of adenoviral particles locally within the vasculature (to the site of vessel wall injury) or within a tissue $(5-7,21)$. Specially modified catheter delivery systems including coated balloons and intravascular stents have been designed in order to achieve high local concentrations of adenovirus within the vasculature (22-24). However, the usefulness of these approaches may be limited within the human coronary circulation due to the high frequency of side branches. Moreover, such catheter delivery systems do not restrict transgene expression to specific cell types in the vessel wall. Finally, several groups have reported that the tissue tropism of RDAd can be modified by electrostatically conjugating adenoviral proteins to ligands that can bind specifically to tissue-specific cell surface receptors $(25,26)$. This approach has been used to successfully target RDAd to hepatocytes and hematopoietic progenitor cell lines (26).

The use of tissue-specific transcriptional regulatory elements represents an alternative strategy to restrict adenoviral transgene expression to specific cell lineages or tissues in vivo (20). While theoretically appealing, this strategy is potentially

1. Abbreviations used in this paper: ALT, alanine aminotransferase; AP, alkaline phosphatase; AST, aspartate aminotransferase; CMV, cytomegalovirus; HUVEC, human umbilical vein endothelial cell; PFU, plaque-forming units; RDAd, replication-defective adenoviruses; SMC, smooth muscle cell. 
limited because the adenovirus genome contains multiple highly active transcriptional enhancers that are capable of transactivating a variety of different promoters in multiple cell lineages (15). Such a targeting strategy is particularly problematic in SMCs because of the lack of SMC-specific transcriptional regulatory elements that function in vivo. This is due primarily to the lack of definitive markers of this muscle cell lineage and the poorly understood and diverse embryological origin(s) of these cells (for review see reference 27).

$\mathrm{SM} 22 \alpha$ is an early marker of the SMC lineage that is expressed at high levels exclusively in visceral and vascular SMCs during postnatal development (28-30). We have shown that the 441-bp SM22 $\alpha$ promoter activates transcription exclusively in arterial SMCs during postnatal development in transgenic mice (31). To test whether the arterial SMC-specific SM $22 \alpha$ promoter could be used to restrict the activity of an RDAd to SMCs, we generated AdSM22-lacZ, an RDAd containing the bacterial lac $Z$ reporter gene under the transcriptional control of the 441-bp murine SM22 $\alpha$ promoter. In the studies described in this report, we demonstrate, that after in vitro infection, the lac $Z$ reporter gene encoded by AdSM22-lacZ is expressed exclusively in SMCs. Moreover, the SMC specificity of the AdSM22-lacZ virus is maintained after intraarterial administration of the virus to an isolated segment of both uninjured and balloon-injured rat carotid arteries. In contrast to previously described adenoviral vectors (18-20), intravenous administration of AdSM22-lacZ does not produce detectable transgene expression in the liver or lung. Finally, we have demonstrated that AdSM22-lacZ also programs lacZ expression in visceral as well as vascular SMCs in vivo. These studies demonstrate that RDAd-encoded transgene expression can be restricted to the SMC lineage by the SM22 $\alpha$ promoter. The demonstration of an efficient gene delivery system targeted specifically to SMCs provides a novel means to deliver recombinant gene products to either vascular or visceral SMCs. As such, SM $22 \alpha$ promoter-driven RDAd may prove useful in the treatment of vascular proliferative diseases as well as visceral SMC-related disorders.

\section{Methods}

Plasmids and replication-defective adenoviral vectors. The pAdSM22 plasmid was generated by subcloning the 441-bp murine SM22 $\alpha$ promoter (30) into ClaI ( $5^{\prime}$ end)/HindIII ( $3^{\prime}$ end)-digested pAdEF1(KN) plasmid (32). The pAdSM22-lacZ plasmid was generated by subcloning the HindIII (5' end)/BgIII ( $3^{\prime}$ end)-linked bacterial lac $Z$ reporter gene into the HindIII/BamHI-digested pAdSM22 plasmid. The AdSM22-lacZ adenovirus encoding the bacterial lac $Z$ reporter gene under the transcriptional control of the murine SM22 $\alpha$ promoter and the human 4F2 heavy chain transcriptional enhancer (33) was generated by recombination in 293 cells between the pAdSM22-lacZ plasmid DNA and E1- and E3-deleted Ad5Sub360 genomic DNA digested with $\mathrm{XbaI}$ and $\mathrm{ClaI}$ as described previously (34). The structure of this virus was confirmed by Southern blot analyses. The AdCMVlacZ RDAd encoding the bacterial lac $Z$ reporter gene under the transcriptional control of the cytomegalovirus (CMV) immediate early gene promoter/enhancer has been described previously (35). Recombinant viruses were plaque purified three times to avoid contamination with replication-competent virus. High titer adenoviral stocks were prepared by infecting 293 cells with $2-5$ plaque-forming units (PFU) of virus per cell as described previously (34). Titers of each cesium chloride purified viral stock were determined from the absorbance at $260 \mathrm{~nm}\left(1\right.$ absorbance unit $\left.=10^{10} \mathrm{PFU} / \mathrm{ml}\right)$ and were confirmed by plaque assay as described previously (34).
Cell culture and in vitro infection with RDAd. Primary rat aortic SMCs were isolated from 12-16-wk-old Sprague-Dawley rats and grown as described previously (6). Virtually all cells stain positive for expression of SM- $\alpha$-actin when isolated using this technique (30). In all experiments, only third passage primary rat aortic SMCs were used. Immortalized rat vascular A7r5 SMCs, passage 4 human umbilical vein endothelial cells (HUVECs), and mouse NIH 3 T3 fibroblasts were grown as described previously (31). Cells were placed in medium containing $2 \%$ FBS and infected with either 1,10 , or 100 PFU/cell of purified adenoviral stocks. After infection, cells were washed in PBS and placed in growth medium containing 10\% FBS. $72 \mathrm{~h}$ after infection, cells were harvested for preparation of DNA and RNA, or were fixed and stained for $\beta$-galactosidase activity with $\mathrm{X}$-gal as described (36). The unstained and blue-stained $\left(\beta\right.$-gal $\left.{ }^{+}\right)$cells from 10 representative high-power fields were counted in each section and the percentage of $\beta$-gal ${ }^{+}$cells was calculated. The data are expressed as percent $\beta$-galactosidase positive cells \pm SEM. All experiments involving animals were approved by the University of Chicago Committee on Animal Care and Use. The Sprague-Dawley rats were housed and cared for according to National Institutes of Health guidelines in the A.J. Carlson Animal Research Facility at the University of Chicago.

Northern and Southern blot analyses. Southern and Northern blot analyses were performed as described previously (37). PCR-generated 485-bp bacterial lac $Z$ probe (which corresponds to bp 962-1448 in the $\mathrm{pCMV} \beta$ plasmid; Clontech, Palo Alto, CA) was radiolabeled and used for the Southern and Northern blot analyses. Quantitative image analyses were performed using a PhosphorImager (Molecular Dynamics, Sunnyvale, CA).

Intraarterial administration of RDAd in uninjured and ballooninjured rat carotid arteries. After induction of anesthesia and intubation, the left and right carotid arteries of adult Sprague-Dawley rats were isolated and a balloon injury was created by dilatation with a $2 \mathrm{~F}$ Fogarty catheter as described previously (6). A 24-gauge intravenous catheter was introduced into the lumen of uninjured or ballooninjured arterial segments and $2 \times 10^{9} \mathrm{PFU}$ of AdSM22-lacZ or AdCMVlacZ was instilled into the isolated arterial segment for $5 \mathrm{~min} .7 \mathrm{~d}$ after infection, rats were killed and the isolated segments of carotid artery were removed, fixed in $1.25 \%$ glutaraldehyde, and stained for $\beta$-galactosidase activity with X-gal as described previously (36). Photomicroscopy was performed using Kodak EPT 160 film and a Zeiss Axiophot microscope.

Intravenous administration of RDAd. 12-16-wk-old Sprague-Dawley rats were injected intravenously with $10^{9}$ or $10^{10}$ PFU of AdSM22lacZ or AdCMV-lacZ, respectively. Liver function tests were performed on serum samples obtained $7 \mathrm{~d}$ after infection using Kodak DT60II and DTSCII automated analyzers. To determine the significance of alterations in liver function tests observed between control, AdSM22-lacZ-infected, and AdCMV-lacZ-infected rats, Student's $t$ tests were performed. $7 \mathrm{~d}$ after injection, rats were killed and the injected tissue, as well as the liver, lung, kidney, and carotid arteries were isolated, washed, fixed, and stained for $\beta$-galactosidase activity with X-gal as described previously (36).

Direct injection of RDAd into visceral SMCs and skeletal muscle. Direct injection of AdSM22-lacZ into SMC-containing tissues and skeletal muscle was performed after induction of anesthesia and intubation as described above. $10^{9} \mathrm{PFU}$ of the AdSM22-lacZ virus was injected directly with a 30-gauge needle into the wall of the ureter, the bladder wall, or intramuscularly. The site of each injection was marked by a suture. $7 \mathrm{~d}$ after injection, the sites of injection were isolated, fixed, and stained for $\beta$-galactosidase activity as described (36).

\section{Results}

Transgene expression from AdSM22-lacZ is restricted to SMCs in vitro. The 441-bp murine SM22 $\alpha$ promoter has been shown previously to program arterial SMC-specific gene expression 
in transgenic mice $(31,38,39)$. To test whether the SM22 $\alpha$ promoter could be used to restrict the expression of a recombinant gene product encoded by an RDAd to SMCs, we constructed an RDAd (AdSM22-lacZ) containing the bacterial lac $Z$ reporter gene under the transcriptional control of the murine SM $22 \alpha$ promoter (Fig. $1 A$, top). In the experiments described below, the activity of AdSM22-lacZ was compared with that of the control virus, AdCMV-lacZ (32), in which the bacterial lac $Z$ reporter gene is under the transcriptional control of the ubiquitously active CMV immediate early gene promoter/enhancer (Fig. 1 A, bottom).
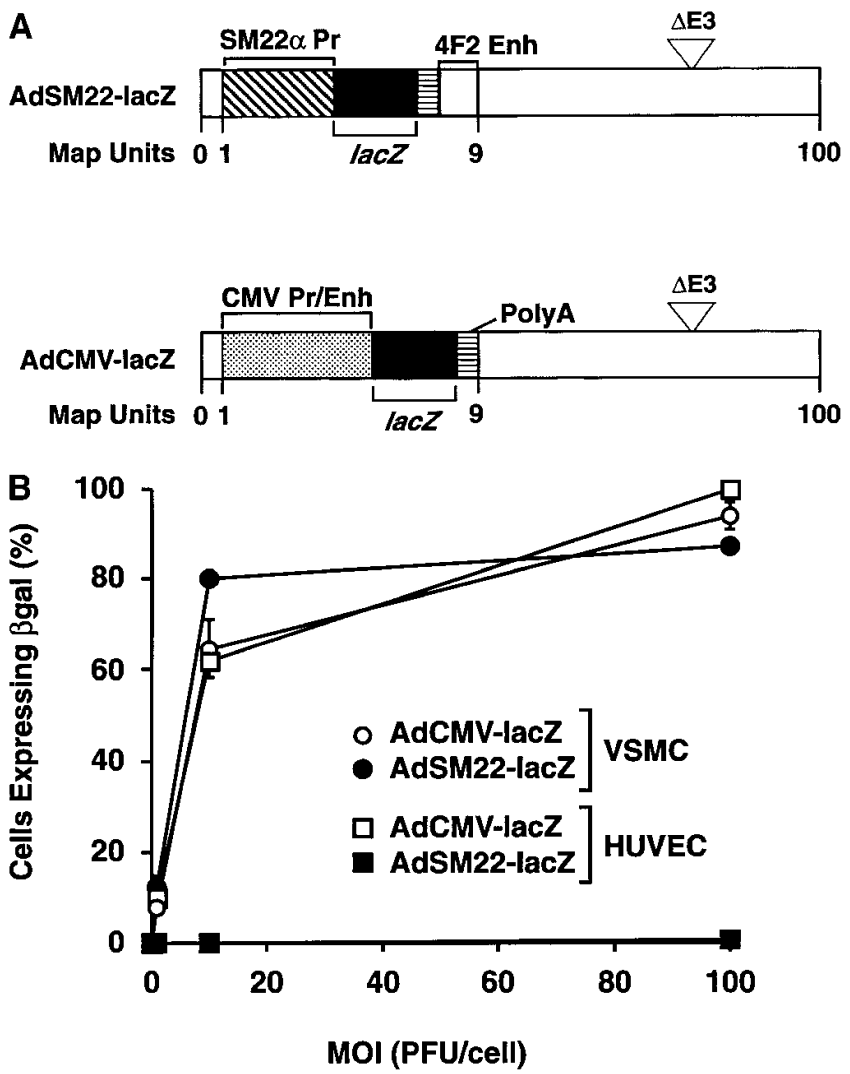

Figure 1. Structure and in vitro expression of AdSM22-lacZ. (A) Schematic representations of the AdSM22-lacZ and AdCMV-lacZ adenoviral vectors. The AdSM22-lacZ vector (top) encodes the bacterial lacZ reporter gene (black box) and bovine growth hormone polyadenylation signal (box with horizontal lines) under the transcriptional control of the 441-bp murine SM22 $\alpha$ promoter (box with diagonal lines) and the 450-bp human 4F2 transcriptional enhancer (white box). The E1 and E3 regions of the Ad5Sub360 adenoviral genome were deleted $(\Delta \mathrm{E} 3)$, rendering the virus replication-defective. The AdCMV-lacZ control virus encodes the lacZ reporter gene (black box) under the transcriptional control of the CMV immediate early gene promoter enhancer (box filled with dots). (B) Comparison of the activity of AdSM22-lacZ and the AdCMV-lacZ control virus in primary cultures of rat aortic smooth muscle cells $(V S M C)$ and HUVECs. Primary cultures of VSMCs or HUVECs were infected with 1,10, and $100 \mathrm{PFU}$ of either AdSM22-lacZ (black squares and black circles) or AdCMV-lacZ (open squares and open circles) and the percentage of cells expressing $\beta$-galactosidase activity was quantitated $72 \mathrm{~h}$ after infection. Data are expressed as the mean percentage of cells expressing $\beta$ gal activity \pm SEM.
To assess the activity of AdSM22-lacZ in cells transduced in vitro, replicate cultures of primary rat aortic SMCs were infected with 1, 10, and $100 \mathrm{PFU} /$ cell of either AdSM22-lacZ or AdCMV-lacZ and the fraction of cells expressing histochemically identifiable $\beta$-galactosidase activity (as assessed by blue staining with Xgal) was quantitated. As shown in Fig. $1 B$ and Fig. $2 A, 12,80$, and $88 \%$ of cells expressed the lac $Z$ transgene after infection with 1, 10, and $100 \mathrm{PFU} /$ cell, respectively, of AdSM22-lacZ. The fraction of cells expressing $\beta$-galactosidase was comparable to that observed after infection of replicate cultures with the control AdCMV-lacZ virus (Fig. $1 B$ and Fig. $2 B$ ). Consistent with these findings, 10,70 , and $90 \%$, respectively, of immortalized A7r5 vascular SMCs expressed the lacZ transgene after infection with 1, 10, and $100 \mathrm{PFU} /$ cell of AdSM22-lacZ (Fig. $2 C$ and data not shown). This efficiency of transgene expression was, again, comparable to that observed after infection of this immortalized SMC line with the AdCMVlacZ control virus (Fig. $2 \mathrm{D}$ and data not shown).

To determine whether the SM22 $\alpha$ promoter restricted expression of the $l a c Z$ reporter gene to SMCs, primary HUVECs and NIH 3T3 fibroblasts were infected with AdSM22-lacZ or the AdCMV-lacZ control virus. In contrast to the high efficiency of transgene expression observed after AdSM22-lacZmediated infection of primary and immortalized SMCs (Fig. 1 $B$ and Fig. 2, $A$ and $C$ ), $\beta$-galactosidase activity was not detectable in HUVECs or NIH 3 T3 cells after infection with AdSM22-lacZ (Fig. $1 B$ and Fig. 2, $E$ and $G$ ). In contrast, 10, 60 , and $93 \%$ of HUVECs expressed histochemically detectable $\beta$-galactosidase activity after infection with 1,10 , and 100 PFU, respectively, of the AdCMV-lacZ control virus (Fig. $1 B$ and Fig. $2 F$ ). Similarly, $\sim 50 \%$ of NIH 3 T3 cells expressed detectable $\beta$-galactosidase activity after infection with $100 \mathrm{PFU} /$ cell of the AdCMV-lacZ control virus (Fig. $2 H$ ).

Southern blot analyses of DNA harvested from HUVECs infected $72 \mathrm{~h}$ previously with AdSM22-lacZ demonstrated the presence of the lac $Z$ transgene in these cells (Fig. 3 A, arrow, lanes 4 and 5). The hybridization signal was comparable in intensity to that obtained with DNA harvested from HUVECs infected with the AdCMV-lacZ control virus, thereby confirming efficient infection of these cells by AdSM22-lacZ (Fig. $3 A$, arrow, lanes 2 and 3 ). Of note, the different sizes of the lacZ hybridizing bands seen in this experiment (Fig. 3, arrows) are consistent with the expected patterns of restriction endonuclease digestion of each adenoviral vector with BglII. Despite the fact that AdSM22-lacZ and AdCMV-lacZ both efficiently infected HUVECs, no lacZ transgene mRNA was detected in the AdSM22-lacZ infected HUVECs by Northern blot analysis (Fig. $3 B$, lanes 4 and 5). In contrast, HUVECs infected with the control AdCMV-lacZ virus expressed abundant lac $Z$ mRNA (Fig. 3 B, arrow, lanes 2 and 3). Taken together, these data demonstrated that AdSM22-lacZ programs SMC-specific transgene expression in vitro and confirmed that the lineagerestricted expression of the transgene was regulated at a transcriptional or posttranscriptional level.

Intraarterial administration of the AdSM22-lacZ virus to uninjured and balloon-injured rat carotid arteries. To determine whether the SM22 $\alpha$ promoter could be used to restrict adenovirus-mediated transgene expression to arterial SMCs in vivo, $2 \times 10^{9} \mathrm{PFU}$ of either AdSM22-lacZ or the control AdCMVlacZ virus was introduced into isolated segments of uninjured and balloon-injured rat carotid arteries. As shown in Fig. 4, $A$ and $B$, diffuse blue staining of the vascular endothelium was 
AdSM22-lac Z

\section{$1^{\circ} \mathrm{VSMC}$}

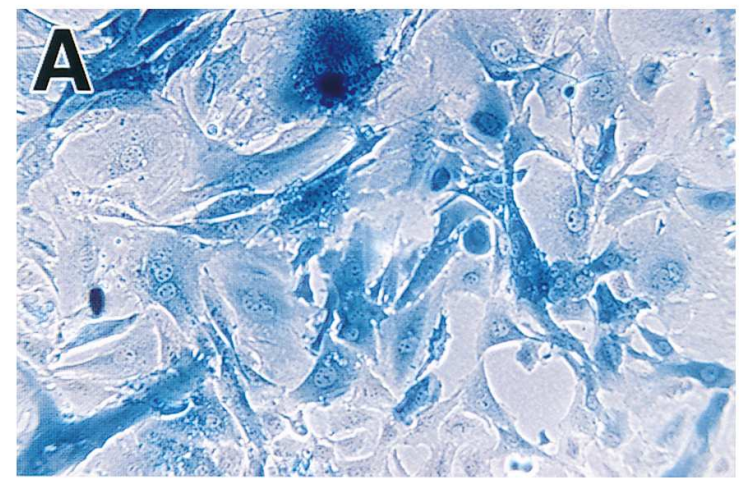

A7r5
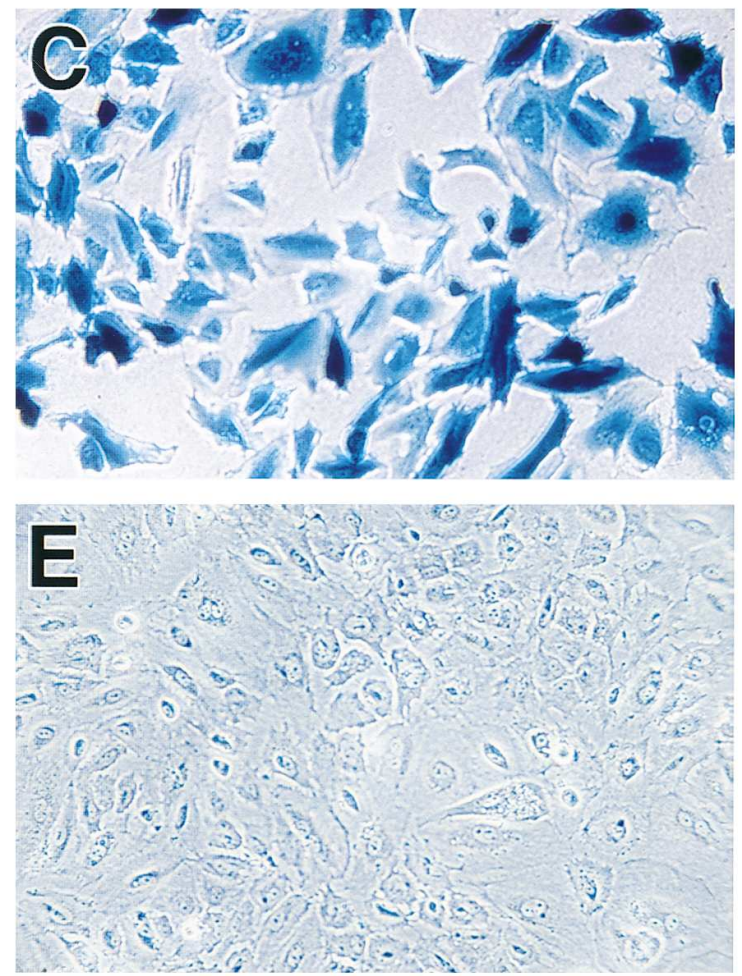

\section{G}

\section{AdCMV-lac Z}
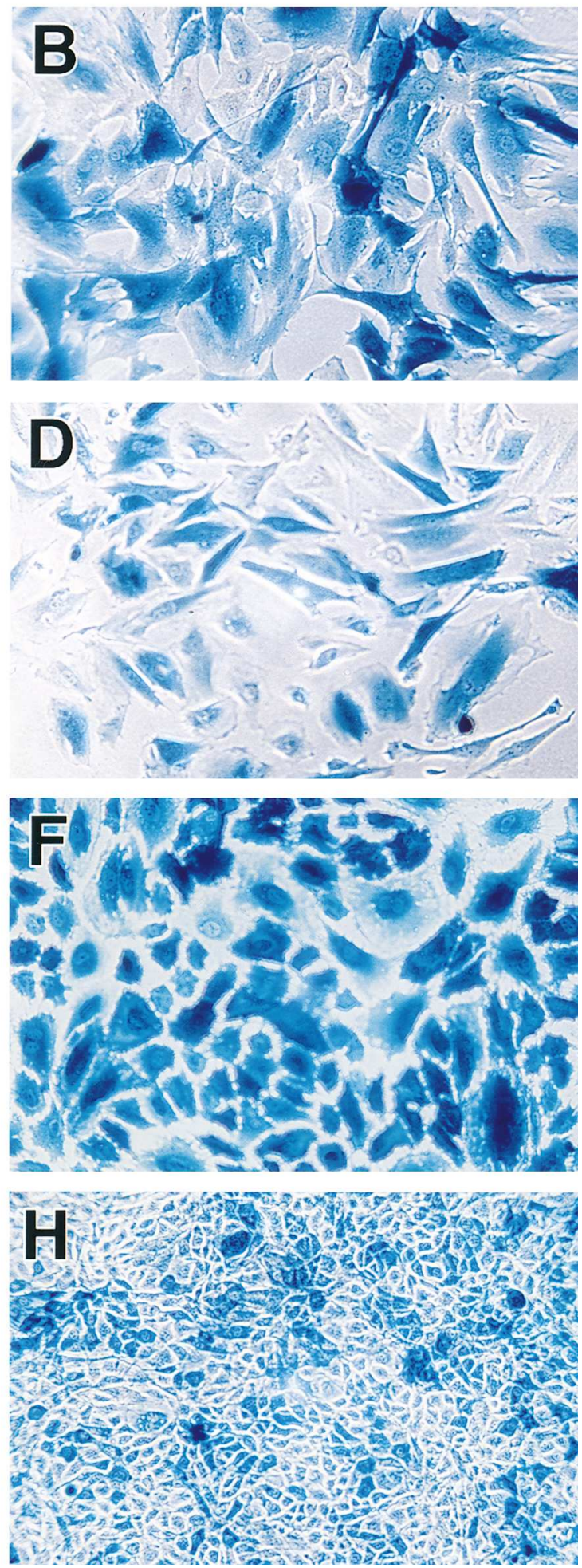

Figure 2. SMC specificity of AdSM22-lacZ after in vitro gene transduction. Replicate cultures of primary rat aortic SMCs $(A$ and $B)$, A7r5

SMCs $(C$ and $D)$, HUVECs $(E$ and $F)$, and NIH 3 T3 fibroblasts $(G$ and $H)$ were infected with 10 PFU/cell of either the AdSM22-lacZ virus $(A$, $C, E$, and $G)$, or the AdCMV-lacZ control virus $(B, D, F$, and $H)$. $72 \mathrm{~h}$ after infection, cells were fixed and stained for $\beta$-galactosidase activity as described in Methods. Expression of the lac $Z$ reporter gene encoded within the AdSM22-lacZ virus was observed in primary rat aortic SMCs $(A)$ and the immortalized SMC line A7r5 $(C)$, but not in HUVECs $(E)$ or NIH 3 T3 cells $(G)$. In contrast, the lac $Z$ reporter gene encoded within the AdCMV-lacZ control virus was expressed in both SMCs and non-SMCs. 

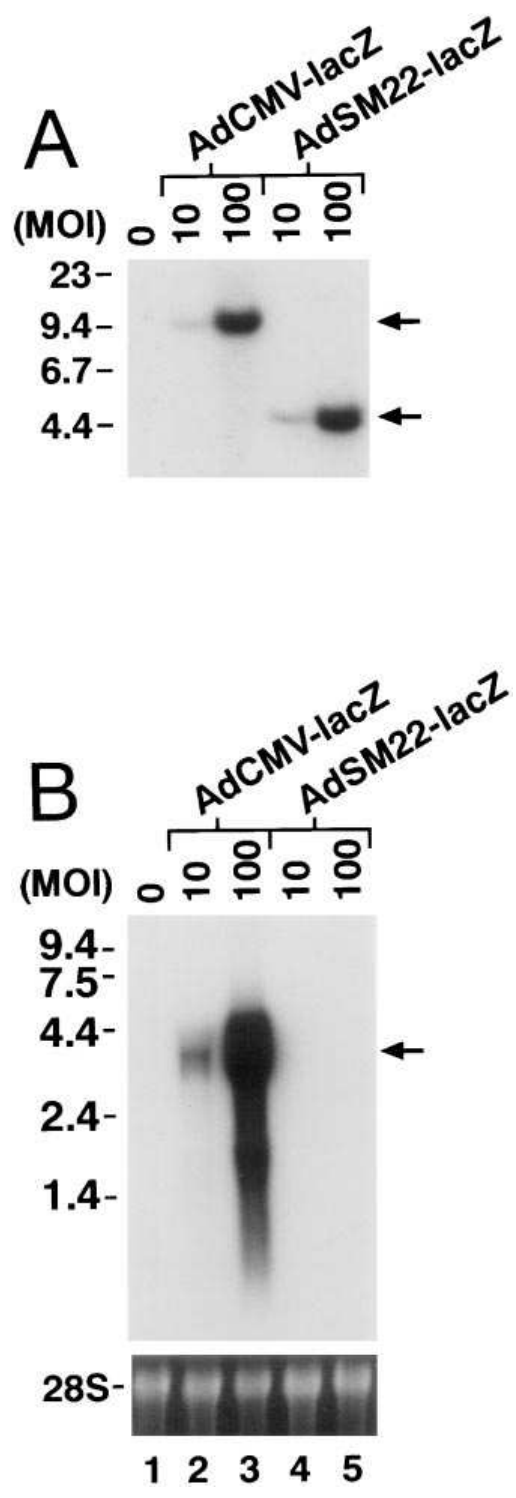

tion signal demonstrated in cells infected with AdCMV-lacZ is indicated with an arrow to the right of the blot. Size markers in kilobases are shown to the left of the blot. The bottom panel shows the ethidium bromide-stained gel before membrane transfer. The position of the 28S ribosomal RNA band is indicated.

observed $7 \mathrm{~d}$ after administration of the control AdCMV-lacZ virus into the uninjured rat carotid artery (Fig. 4 B, arrowheads). In addition, rare cells within the adventitia also stained blue (Fig. $4 A$ ). In contrast, when AdSM22-lacZ was introduced into the uninjured rat carotid artery, $\beta$-galactosidase activity was not observed within either endothelial or adventitial cells (Fig. 4 C). However, rare lacZ-expressing SMCs were observed in the superficial (abluminal) layer of the tunica media (Fig. $4 \mathrm{D}$, arrowhead). These data suggested that the SMC specificity of AdSM22-lacZ transgene expression is maintained after intraarterial administration of AdSM22-lacZ into an isolated segment of the uninjured rat carotid artery.

To determine the cell specificity of transgene expression in balloon-injured rat carotid arteries, $2 \times 10^{9} \mathrm{PFU}$ of AdSM22lacZ was instilled into an isolated segment of the rat carotid ar- tery for 5 min immediately after balloon injury (Fig. 4, $E$ and $F) .7 \mathrm{~d}$ after infection, the injured arterial segments were isolated and the pattern of $\beta$-galactosidase expression was compared with that observed in the uninfected balloon-injured contra lateral artery. In contrast to the low level $\beta$-galactosidase activity observed in the uninjured carotid artery infected with the AdSM22-lacZ virus, higher efficiency gene transfer was achieved in these balloon-injured arterial segments (compare Fig. 4, $C$ and $E$ ). The majority of the SMCs expressing $\beta$-galactosidase activity were located within the tunica media (Fig. $4 F$, black arrowheads). In addition, rare cells within the neointima also stained light blue (Fig. $4 F$, open arrowhead). Consistent with previous reports, gene transfer was preferentially observed in the SMCs underlying the site of neointimal proliferation (Fig. 4 E). Finally, it is noteworthy that lac Z transgene expression was not observed in endothelial cells at the margins of the vessel wall injury, where endothelial cells remained intact (data not shown). Taken together, these data demonstrated that the AdSM22-lacZ virus maintains its SMCspecific pattern of transgene expression after intraarterial administration and that it can be used to efficiently transduce arterial SMCs in the balloon-injured rat carotid artery.

Expression of the lac $Z$ reporter gene after intravenous injection of AdSM22-lacZ. Intravenous administration of RDAd results in high level gene transfer to the liver and lung, thereby potentially limiting the utility of these viruses in some clinical settings (18-20). To determine whether the SMC-specific pattern of expression of the lac $Z$ reporter gene expression programmed by the AdSM22-lacZ virus would be maintained after intravenous administration, $10^{10} \mathrm{PFU}$ of either AdSM22lacZ or the control AdCMV-lacZ virus was injected intravenously into adult Sprague-Dawley rats and representative sections from the livers and lungs of these rats (as well as other tissues) were stained for $\beta$-galactosidase activity. lac $Z$ expression was observed throughout the livers of rats infected with the AdCMV-lacZ control virus (Fig. 5 E). In addition, focal patches of $\beta-\mathrm{gal}^{+}$cells were observed within perivascular regions of the lung of AdCMV-lacZ-infected rats (Fig. 5 F). In contrast, $7 \mathrm{~d}$ after infection with AdSM22-lacZ, histological sections of both the liver and lung (Fig. 5, $C$ and $D$ ) were indistinguishable from those obtained from uninfected control rats (Fig. 5, $A$ and $B$ ). These data suggest that in contrast to RDAd containing virally driven and/or ubiquitously active transcriptional regulatory elements, AdSM22-lacZ restricts transgene expression to SMCs after intravenous administration.

To determine whether intravenous administration of AdSM22-lacZ caused abnormalities in liver function despite the finding that the lac $Z$ reporter gene encoded by this virus was not expressed in this tissue (see Fig. $5 C$ ), adult SpragueDawley rats were injected intravenously with $10^{10} \mathrm{PFU}$ of the AdSM22-lacZ virus and liver function tests were performed on serum samples obtained $7 \mathrm{~d}$ after infection. No statistically significant elevations in serum alkaline phosphatase (AP), alanine aminotransferase (ALT), aspartate aminotransferase (AST), $\gamma$-glutamyltranspeptidase, total bilirubin, total protein, and albumin were observed in rats infected with the AdSM22lacZ virus (Table I) $(P>0.05)$. However, small, but consistent, elevations in the mean serum concentrations of ALT, AST, and AP were observed (Table I). In contrast, statistically significant elevations in ALT and AST serum concentrations were observed $7 \mathrm{~d}$ after intravenous administration of $10^{10}$ PFU of the AdCMV-lacZ control virus $(P<0.05)$. In addition, 

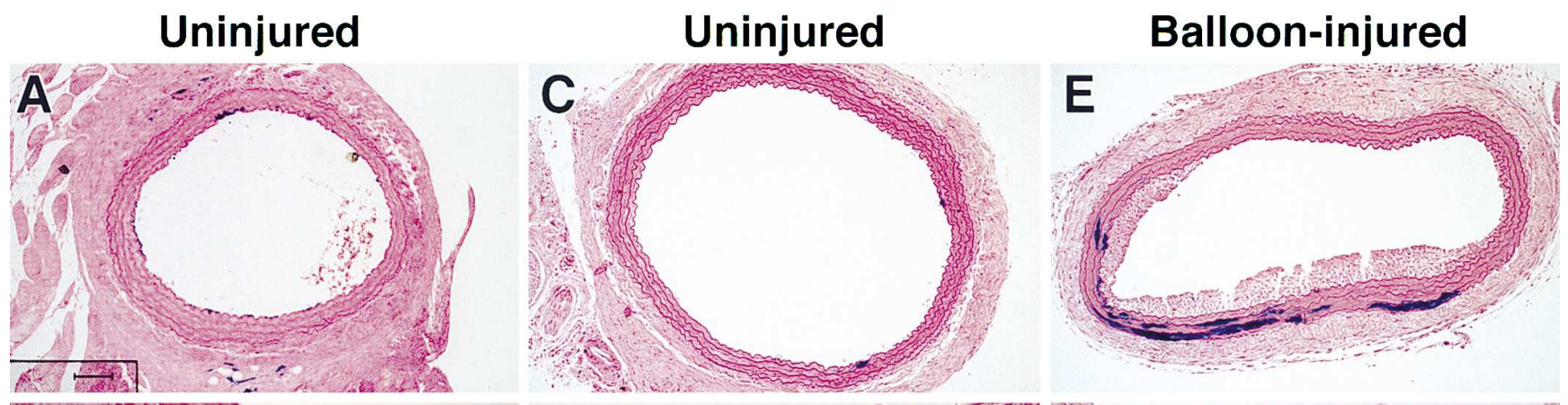

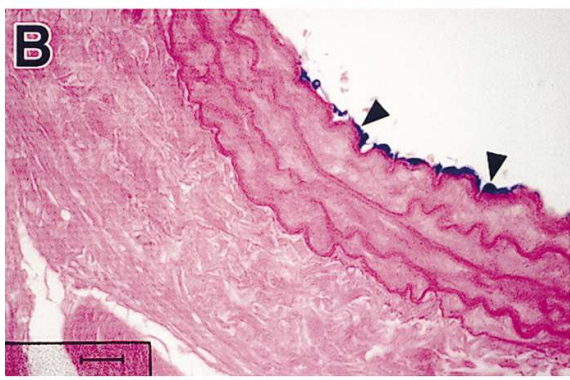

AdCMV-lacZ

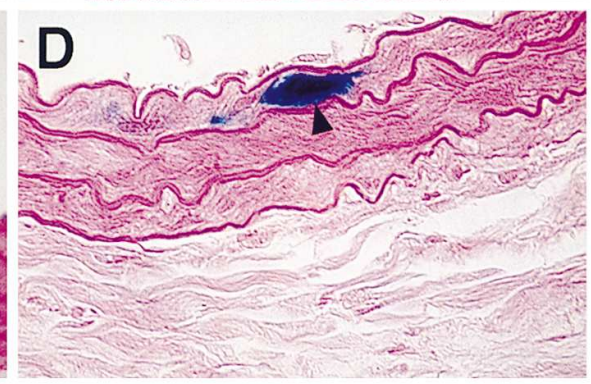

AdSM22-lacZ

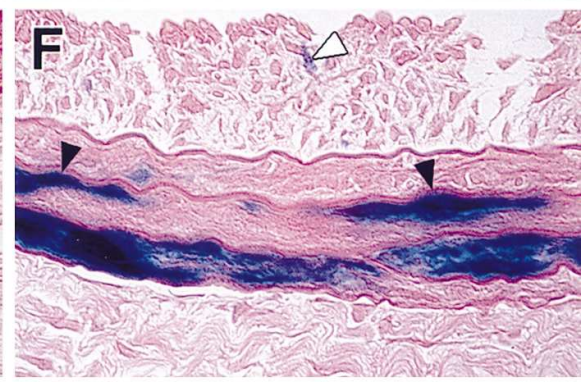

AdSM22-lacZ

Figure 4. lacZ transgene expression in uninjured and balloon-injured rat carotid arteries infected with AdSM22-lacZ and AdCMV-lacZ. ( $A-D$ ) Isolated segments of uninjured rat carotid arteries were infected with $10^{9}$ PFU of either AdSM22-lacZ or the control AdCMV-lacZ virus. $7 \mathrm{~d}$ after infection, rats were killed, and the carotid arteries were removed and stained for $\beta$-galactosidase activity. ( $A$ and $B$ ) In uninjured arteries infected with AdCMV-lacZ, $\beta$-galactosidase activity (blue staining) was observed in endothelial cells (black arrowheads) and in rare cells located within the adventitia. ( $C$ and $D$ ) In uninjured arteries infected with the AdSM22-lacZ virus, $\beta$-galactosidase activity was observed in rare SMCs located within the superficial (abluminal) layer of the tunica media. No staining of endothelial cells was observed. ( $E$ and $F$ ) After anesthesia and intubation, the left and right carotid arteries of Sprague-Dawley rats were isolated and injured by dilatation with a Fogarty catheter. A 24gauge intravenous catheter was introduced into the lumen of each isolated arterial segment and $10^{9}$ PFU of AdSM22-lacZ was instilled for 5 min. $\beta$-galactosidase activity was observed in the SMCs located within the superficial and deep layers of the tunica media (black arrowheads) and within rare cells located within the neointima (open arrowhead). Photomicroscopy was performed using a Zeiss Axiophot microscope. Original magnification, $A, C$, and $E, \times 10 ; B, D$, and $F, \times 40$.
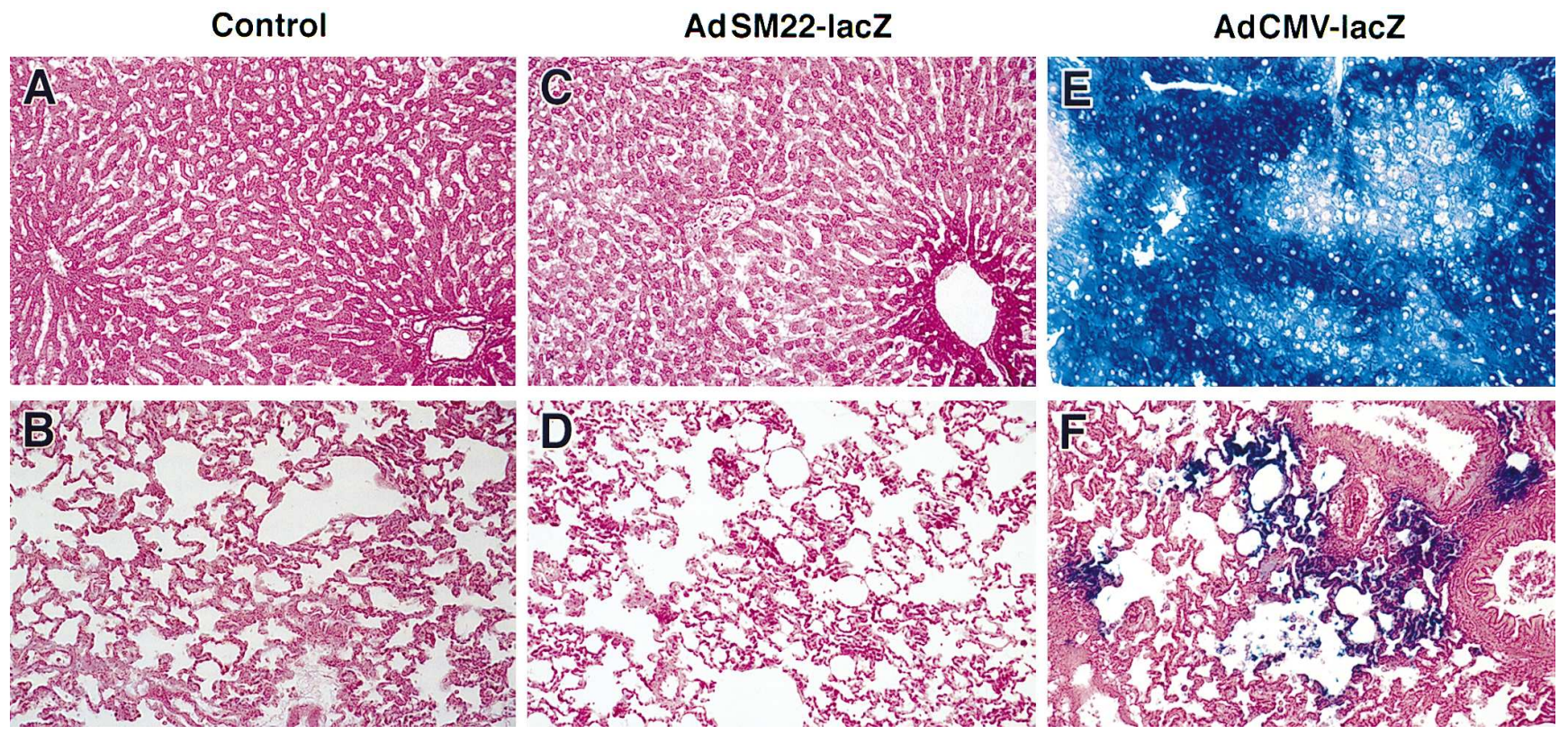

Figure 5. Expression of the lacZ transgene after intravenous administration of AdSM22-lacZ. Adult Sprague-Dawley rats received intravenous injections of $10^{10} \mathrm{PFU}$ of AdSM22-lacZ or AdCMV-lacZ. $7 \mathrm{~d}$ after infection, the rats were killed and their tissues were fixed and stained for $\beta$-galactosidase activity. ( $A, C$, and $E$ ) Representative histological sections through the liver of control rats $(A)$, AdSM22-lacZ-infected rats $(C)$, and AdCMV-lacZ-infected rats $(E)$. $\beta$-galactosidase activity was not observed in control livers or the livers of rats receiving intravenous administration of AdSM22-lacZ virus. In contrast, lac $Z$ expression was demonstrated throughout the livers of rats receiving intravenous injections of AdCMV-lacZ. $(B, D$, and $F)$ Representative histological sections through the lungs of control rats $(B)$, AdSM22-lacZ-infected rats $(D)$, and AdCMV-lacZ-infected rats $(F)$. $\beta$-galactosidase activity was not observed in control lungs or the lungs of rats receiving AdSM22-lacZ. In contrast, focal patches of lacZ-expressing cells were observed in the perivascular regions of AdCMV-lacZ-infected rats. 
increased serum concentrations of AP and bilirubin were observed in rats receiving $10^{10} \mathrm{PFU}$ of the AdCMV-lacZ virus $(P<0.09)$. Thus, intravenous administration of high doses of AdSM22-lacZ did result in mild elevations in liver function tests. However, the liver function test abnormalities were significantly less marked than those observed in rats infected with identical doses of the AdCMV-lacZ control virus.

Expression of $\beta$-galactosidase activity after direct injection of AdSM22-lacZ into visceral smooth muscle and skeletal muscle. To determine whether the lac $Z$ transgene encoded by AdSM22-lacZ was expressed in visceral as well as vascular SMCs, $10^{9}$ PFU of AdSM22-lacZ was injected directly into the wall of the rat ureter and bladder, respectively. $7 \mathrm{~d}$ after infection, histological sections from the sites of injection were stained for $\beta$-galactosidase activity. As shown in Fig. $6 \mathrm{~A}$, dense blue staining was observed throughout the longitudinal and circumferential layers of SMCs within the wall of the ureter (white arrowheads). In contrast, $\beta$-galactosidase activity was not observed within the epithelial cells lining the lumen of the ureter (Fig. 6 A, black arrowheads). After direct injection of AdSM22-lacZ into the bladder mucosa, focal patches of $\beta$-gal ${ }^{+}$SMCs were observed surrounding the site of injection (Fig. $6 \mathrm{~B}$, white arrowheads). In contrast, $\beta$-galactosidase activity was not observed within the bladder epithelium. These data demonstrated that AdSM22-lacZ programs transgene expression in visceral as well as vascular SMCs.

The 441-bp murine SM22 $\alpha$ promoter is active in embryonic skeletal muscle cells and the somites of transgenic mice (31). To determine whether AdSM22-lacZ programs transgene expression in adult skeletal muscle in vivo, $10^{9} \mathrm{PFU}$ of the AdSM22-lacZ virus was injected intramuscularly into the rat rectus abdominus and quadriceps muscles. In contrast to the dense blue staining observed in visceral SMCs after direct injection into the wall of the ureter and bladder (Fig. 6, $A$ and $B), \beta$-galactosidase activity was not observed in either the rectus abdominus (Fig. $6 \mathrm{C}$ ) or quadriceps muscles (Fig. $6 \mathrm{D}$ ). Thus, the lac $Z$ reporter gene encoded by AdSM22-lacZ is expressed exclusively in visceral and vascular SMCs when administered intraarterially, intravenously, or intramuscularly.

\section{Discussion}

SMCs subserve diverse functions in organ systems throughout the body including the modulation of arterial tone, airway resistance, and gut motility. These cells have been implicated in the pathogenesis of a variety of diseases including atherosclerosis, restenosis after percutaneous transluminal coronary angioplasty, and asthma (1-4). Gene transfer using replicationdefective adenoviral vectors has been used to examine the pathogenesis of these diseases (40-43) and also holds promise

Table I. Liver Function in AdSM22-lacZ and AdCMV-lacZ-infected Rats

\begin{tabular}{lccccc}
\hline & ALT & AST & \multicolumn{1}{c}{ AP } & Bilirubin & Albumin \\
\hline Control & $61 \pm 4$ & $97 \pm 8$ & $229 \pm 38$ & $0.1 \pm 0.0$ & $2.9 \pm 0.1$ \\
$10^{10} \mathrm{SM} 22-$ lacZ & $104 \pm 33$ & $137 \pm 21$ & $306 \pm 37$ & $0.1 \pm 0.0$ & $3.7 \pm 0.2$ \\
$10^{10} \mathrm{CMV}-$ lacZ & $153 \pm 16^{*}$ & $166 \pm 21^{*}$ & $442 \pm 103^{\ddagger}$ & $0.3 \pm 0.2^{\ddagger}$ & $3.3 \pm 0.2$
\end{tabular}

Data are expressed as mean \pm SEM. $* P<0.05$ versus control values; ${ }^{\ddagger} P<$ 0.09 versus control values. for their treatment (11). However, adenovirus-mediated gene transfer is potentially limited in both the clinical setting and in animal models of human disease, because of the capacity of these viruses to indiscriminately infect many cell types $(5,15)$. In the studies described in this report, we have generated an RDAd, designated AdSM22-lacZ, which contains the bacterial lac $Z$ reporter gene under the transcriptional control of the SMC-specific SM22 $\alpha$ promoter $(30,31,38)$. We have demonstrated that AdSM22-lacZ programs SMC-specific expression of the $l a c Z$ reporter gene in cultured cells. In addition, we have shown that the SMC specificity of the AdSM22-lacZ virus is maintained in vivo after intraarterial, intramuscular, and intravenous administration. Finally, we have demonstrated that AdSM22-lacZ programs transgene expression in visceral, as well as vascular SMCs in vivo.

To our knowledge, this report represents the first demonstration that the expression of a recombinant gene product encoded by an RDAd can be regulated in a cell lineagerestricted fashion by a transcriptional regulatory element in normal cells in vivo. Previously, we generated adenoviral vectors containing other cell lineage-specific transcriptional regulatory elements and observed that most of these elements lose their cell lineage specificity when tested in vivo in the context of an adenoviral vector (Leiden, J., and M. Parmacek, unpublished observation). Similarly, Arbuthnot et al. (44) reported that RDAd containing transgenes under the transcriptional control of the alpha-fetoprotein (AFP) promoter are capable of mediating cell lineage-restricted gene expression in hepatoma cells, but not in normal liver parenchyma. It is possible that the 441-bp murine SM22 $\alpha$ promoter may contain an insulator element or a locus control region that is capable of protecting it from the activity of cryptic transcriptional regulatory elements located within the adenoviral genome. In this regard it is noteworthy that the 441-bp murine SM22 $\alpha$ promoter is as active as the most potent viral LTRs in SMCs (30), and functions in an SMC-specific fashion both in vitro and in transgenic mice in vivo $(31,38,39)$.

In transgenic mice the 441-bp SM22 $\alpha$ promoter is active in arterial but not visceral SMCs $(29,31)$. Therefore, the demonstration that the lac $Z$ reporter gene encoded by AdSM22-lacZ was expressed in visceral as well as vascular SMCs was somewhat surprising. This difference may reflect the fact that in adenovirus-infected cells DNA remains episomal, whereas in transgenic mice it is integrated into the host genome where its transcriptional activity can be modulated by alterations in chromatin structure. In support of this hypothesis, we have observed high-level transcriptional activity of the SM22 $\alpha$ promoter in primary cultures of bovine tracheal SMCs after transient transfection (Solway, J., and M. Parmacek, unpublished observation). Clearly, caution must be exercised in extrapolating data generated by either transient transfection analyses or in transgenic mice to the expected cell lineage specificity of a transcriptional regulatory element within the context of a replication-defective adenoviral vector.

RDAd are particularly useful tools for studying the molecular pathogenesis of atherosclerosis and other vascular proliferative disorders. Adenoviruses can be delivered in spatially and temporally restricted fashions to the vessel wall in both normal and atherosclerotic vessels in large and small animals $(13,14)$. However, previous studies using these vectors to investigate the pathogenesis of vascular proliferative disorders have not been able to distinguish effects due to alterations in 

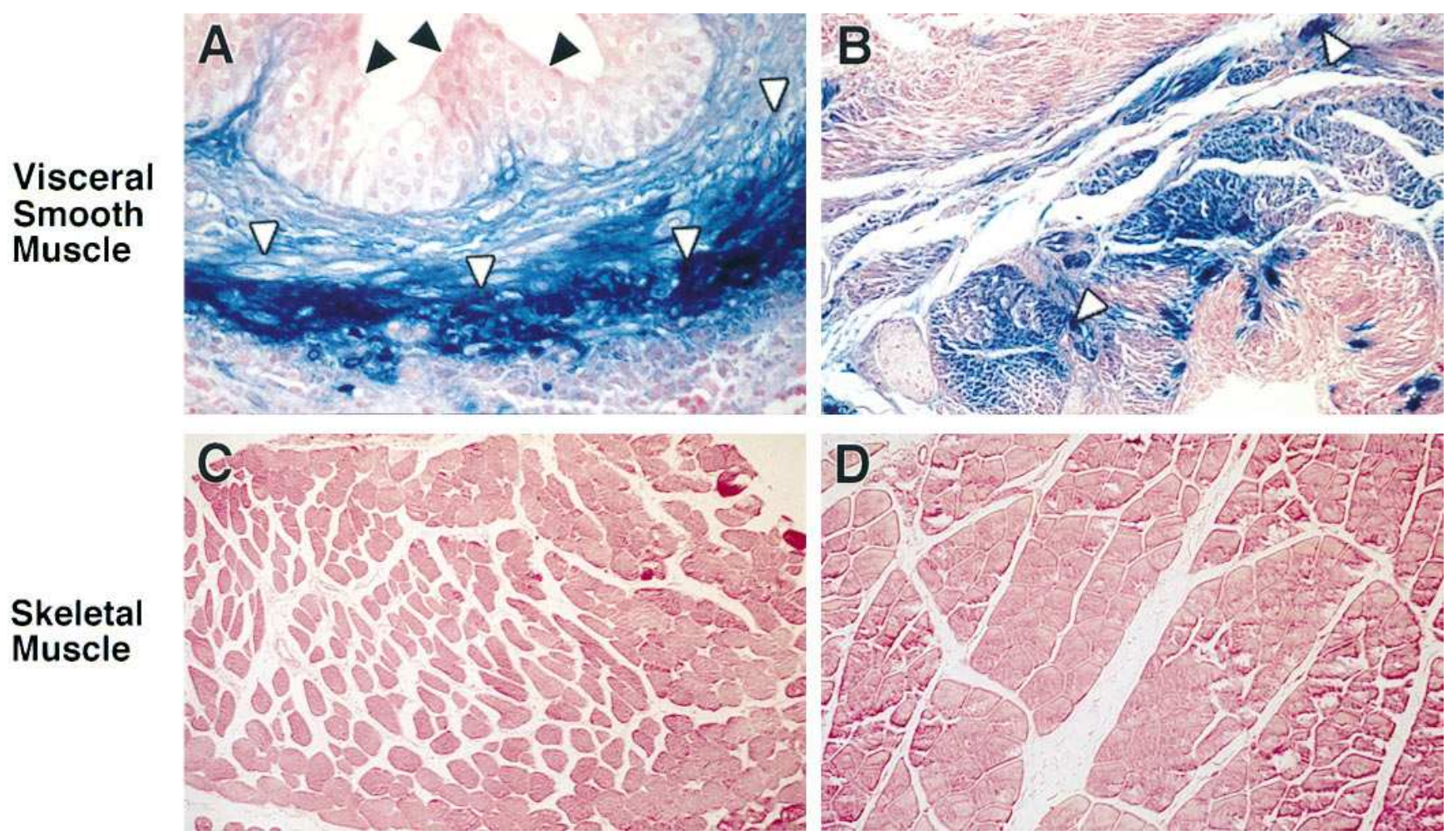

Figure 6. Expression of the lacZ transgene after direct injection of AdSM22-lacZ into visceral smooth muscle and skeletal muscle. Direct injection of the AdSM22-lacZ virus into SMC-containing tissues and skeletal muscle was performed after induction of anesthesia and intubation. $10^{9}$ PFU of AdSM22-lacZ was injected directly with a 30-gauge needle into the wall of the ureter $(A)$, the bladder wall $(B)$, or intramuscularly $(C$ and $D$ ). The site of each injection was marked by a suture. $7 \mathrm{~d}$ after injection, the sites of injection were isolated, fixed, and stained for $\beta$-galactosidase activity. $(A)$ Representative histological section through the ureter injected with AdSM22-lacZ virus. Expression of the lacZ reporter gene by AdSM22-lacZ was observed in the circumferential and longitudinal layers of SMCs within the wall of the ureter (white arrowheads). In contrast, lacZ expression was not observed in the epithelial cells lining the lumen of the ureter (black arrowheads). (B) Representative histological section though the bladder injected with AdSM22-lacZ. lacZ expression (white arrowheads) was observed within SMCs in the bladder wall. lac $Z$ expression was not observed in the underlying bladder epithelium. ( $C$ and $D$ ) Representative histological sections though the rectus abdominus $(C)$ and quadriceps $(D)$ muscles injected intramuscularly with AdSM22-lacZ. In contrast to the $\beta$-galactosidase activity observed within visceral SMCs $(A$ and $B)$, blue-stained skeletal muscle cells were not observed at the sites of intramuscular injection.

vascular SMC gene expression from those resulting from transgene expression in endothelial or adventitial cells. In this respect, RDAd-containing transgenes under the control of the SM22 $\alpha$ promoter will allow us to determine directly the effects of SMC-specific transgene expression on vascular SMC proliferation and neointima formation. In addition, because SM22 $\alpha$ containing RDAd program transgene expression in visceral SMCs, these viruses may also be useful to examine the pathogenesis and treatment of diseases mediated by visceral SMCs. Examples of such diseases include asthma, achalasia, leiomyosarcomas, irritable bowel syndrome, and uterine leiomyomas.

Although the efficacy of RDAd has been established in both large and small animal models of vascular proliferative disease $(5-7,12,21)$, safety concerns persist due to the capacity of these viruses to infect most cells and tissues. In this regard, SM22 $\alpha$ promoter-driven adenoviruses may prove advantageous as vehicles to deliver therapeutic genes to the vessel wall for the treatment of vascular proliferative disorders. The lack of cytotoxic or cytostatic transgene expression in the endothelial cells at the margins of the arterial injury should, in theory, promote more rapid reendothelialization of the injured vessel by facilitating the proliferation and migration of adjacent en- dothelial cells (5). Of equal importance, the potential for systemic toxicity resulting from ectopic expression of potentially toxic adenovirus-encoded transgenes in other tissues and organs should be reduced by use of the SMC-specific SM22 $\alpha$ promoter. Finally, recent reports have demonstrated that much of the immunogenicity of adenovirus-infected cells is due to cellular and humoral immune responses directed against foreign transgene proteins (18). By restricting ectopic expression of adenovirus-encoded transgenes in non-SMC containing tissues, AdSM22 viruses may also reduce the immune-mediated damage to organs such as the liver and the lung after intentional or inadvertent systemic administration of the vector. Our finding of decreased hepatic toxicity after intravenous administration of high dose AdSM22-lacZ supports this hypothesis.

\section{Acknowledgments}

We thank Alex Morla for providing HUVECs. We thank Julian Solway for communicating unpublished data. We thank Lisa Gottschalk for expert preparation of figures and Amy Murphy for secretarial assistance. 
This work was supported in part by Public Health Service grants R01HL56915 to M.S. Parmacek and R01-AR42885 to J.M. Leiden and by the Falk Charitable Trust. M.S. Parmacek is an Established Investigator of the American Heart Association.

\section{References}

1. Ross, R. 1993. The pathogenesis of atherosclerosis: a perspective for the 1990s. Nature (Lond.). 362:801-809.

2. Nikol, S., T.Y. Huehns, and B. Hofling. 1996. Molecular biology and post-angioplasty restenosis. Atherosclerosis. 123:17-31.

3. Bauters, C. 1995. Gene therapy for cardiovascular diseases. Eur. Heart J. 16:1166-1168.

4. James, A.L., P.D. Pare, and J.C. Hogg. 1989. The mechanics of airway narrowing in asthma. Am. Rev. Respir. Dis. 139:242-246.

5. Ohno, T., D. Gordon, H. San, V.J. Pompili, M.J. Imperiale, G.J. Nabel, and E.G. Nabel. 1994. Gene therapy for vascular smooth muscle cell proliferation after arterial injury. Science (Wash. DC). 265:781-784.

6. Chang, M.W., E. Barr, J. Seltzer, Y.-Q. Jiang, G.J. Nabel, E.G. Nabel, M.S. Parmacek, and J.M. Leiden. 1995. Cytostatic gene therapy for vascular proliferative disorders with a constitutively active form of the retinoblastoma gene product. Science (Wash. DC). 267:518-522.

7. Guzman, R.J., E.A. Hirschowitz, S.L. Brody, R.G. Crystal, S.E. Epstein, and T. Finkel. 1994. In vivo suppression of injury-induced vascular smooth muscle cell accumulation using adenovirus-mediated transfer of the herpes simplex virus thymidine kinase gene. Proc. Natl. Acad. Sci. USA. 91:10732-10736.

8. Flugelman, M.Y. 1995. Inhibition of intravascular thrombosis and vascular smooth muscle cell proliferation by gene therapy. Thromb. Haemostasis. 74: $406-410$

9. Muhlhauser, J., M.J. Merrill, R. Pili, H. Maeda, M. Bacic, B. Bewig, A. Passaniti, N.A. Edwards, R.G. Crystal, and M.C. Capogrossi. 1995. VEGF165 expressed by a replication-deficient recombinant adenovirus vector induces angiogenesis in vivo. Circ. Res. 77:1077-1086.

10. Nabel, E.G. 1995. Gene therapy for vascular diseases. Atherosclerosis. 118(Suppl.):S51-S56.

11. Nabel, E.G. 1995. Gene therapy for cardiovascular disease. Circulation. 91:541-548.

12. Yang, Z.Y., R.D. Simari, N.D. Perkins, H. San, D. Gordon, G.J. Nabel, and E.G. Nabel. 1996. Role of the p21 cyclin-dependent kinase inhibitor in limiting intimal cell proliferation in response to arterial injury. Proc. Natl. Acad. Sci. USA. 93:7905-7910.

13. French, B.A., W. Mazur, N.M. Ali, R.S. Geske, J.P. Finnigan, G.P. Rodgers, R. Roberts, and A.E. Raizner. 1994. Percutaneous transluminal in vivo gene transfer by recombinant adenovirus in normal porcine coronary arteries, atherosclerotic arteries, and two models of coronary restenosis. Circulation. 90:2402-2413.

14. Simari, R.D., H. San, M. Rekhter, T. Ohno, D. Gordon, G.J. Nabel, and E.G. Nabel. 1996. Regulation of cellular proliferation and intimal formation following balloon injury in atherosclerotic rabbit arteries. J. Clin. Invest. 98: $225-235$.

15. Haddada, H., L. Cordier, and M. Perricaudet. 1995. Gene therapy using adenovirus vectors. Curr. Top. Microbiol. Immunol. 199:297-306.

16. Simons, M., E.R. Edelman, J.L. DeKeyser, R. Langer, and R.D. Rosenberg. 1992. Antisense c-myb oligonucleotides inhibit intimal arterial smooth muscle cell accumulation in vivo. Nature (Lond.). 359:67-70.

17. Morishita, R., G.H. Gibbons, K.E. Ellison, M. Nakajima, H. von der Leyen, L. Zhang, Y. Kaneda, T. Ogihara, and V.J. Dzau. 1993. Antisense oligonucleotides directed at cell cycle regulatory genes as strategy for restenosis therapy. Trans. Assoc. Am. Phys. 106:54-61.

18. Kashyap, V.S., S. Santamarina-Fojo, D.R. Brown, C.L. Parrott, D. Applebaum-Bowden, S. Meyn, G. Talley, B. Paigen, N. Maeda, and H.B. Brewer, Jr. 1995. Apolipoprotein E deficiency in mice: gene replacement and prevention of atherosclerosis using adenovirus vectors. J. Clin. Invest. 96:1612-1620.

19. Johns, D.C., H.B. Nuss, N. Chiamvimonvat, B.M. Ramza, E. Marban, and J.H. Lawrence. 1995. Adenovirus-mediated expression of a voltage-gated potassium channel in vitro (rat cardiac myocytes) and in vivo (rat liver). A novel strategy for modifying excitability. J. Clin. Invest. 96:1152-1158.

20. Miller, N., and R. Vile. 1995. Targeted vectors for gene therapy. FASEB (Fed. Am. Soc. Exp. Biol.) J. 9:190-199.

21. Chang, M.W., T. Ohno, D. Gordon, M.M. Lu, G.J. Nabel, E.G. Nabel, and J.M. Leiden. 1995. Adenovirus mediated transfer of the herpes simplex virus thymidine kinase gene inhibits vascular smooth muscle cell proliferation and neointima formation following balloon injury of the rat carotid artery. Mol. Med. 1:172-181.

22. March, K.L., J.E. Madison, and B.C. Trapnell. 1995. Pharmacokinetics of adenoviral vector-mediated gene delivery to vascular smooth muscle cells: modulation by poloxamer 407 and implications for cardiovascular gene therapy. Hum. Gene Ther. 6:41-53.

23. Rajasubramanian, G., R.S. Meidell, C. Landau, M.L. Dollar, D.B. Holt, J.E. Willard, M.D. Prager, and R.C. Eberhart. 1994. Fabrication of resorbable microporous intravascular stents for gene therapy applications. ASAIO (Am. Soc. Artif. Intern. Organs) Trans. 40:M584-M589.

24. Kito, H., T. Suzuki, S. Nagahara, Y. Nakayama, Y. Tsutsui, N. Isutsui, N. Nakajima, and T. Matsuda. 1994. A total delivery system of genetically engineered drugs or cells for diseased vessels. Concept, materials, and fabricated prototype device. ASAIO (Am. Soc. Artif. Intern. Organs) Trans. 40:M260M266.

25. Krasnykh, V.N., G.V. Mikheeva, J.T. Douglas, and D.T. Curiel. 1996. Generation of recombinant adenovirus vectors with modified fibers for altering viral tropism. J. Virol. 70:6839-6846.

26. Schwarzenberger, P.. S.E. Spence, J.M. Gooya, D. Michiel, D.T. Curiel, F.W. Ruscetti, and J.R. Keller. 1996. Targeted gene transfer to human hematopoietic progenitor cell lines through the c-kit receptor. Blood. 87:472-478.

27. Owens, G.K. 1995. Regulation of differentiation of vascular smooth muscle cells. Physiol. Rev. 75:487-517.

28. Duband, J.L., M. Gimona, M. Scatena, S. Sartore, and J.V. Small. 1993. Calponin and SM22 as differentiation markers of smooth muscle: spatiotemporal distribution during avian embryonic development. Differentiation. 55:1-11.

29. Li, L., J.M. Miano, P. Cserjesi, and E.N. Olson. 1996. SM22 $\alpha$, a marker of adult smooth muscle, is expressed in multiple myogenic lineages during embryogenesis. Circ. Res. 78:188-195.

30. Solway, J., J. Seltzer, F.F. Samaha, S. Kim, L.E. Alger, Q. Niu, E.E. Morrisey, H.S. Ip, and M.S. Parmacek. 1995. Structure and expression of a smooth muscle cell-specific gene, SM22 $\alpha$. J. Biol. Chem. 270:13460-13469.

31. Kim, S., H.S. Ip, M.M. Lu, C. Clendenin, and M.S. Parmacek. 1997. A serum response factor-dependent transcriptional regulatory program identifies distinct smooth muscle cell sublineages. Mol. Cell. Biol. 17:2266-2278

32. Tripathy, S.K., E. Goldwasser, M.M. Lu, E. Barr, and J.M. Leiden. 1994. Stable delivery of physiologic levels of recombinant erythropoietin to the systemic circulation by intramuscular injection of replication-defective adenovirus. Proc. Natl. Acad. Sci. USA. 91:11557-11561.

33. Karpinski, B.A., L.H. Yang, P. Cacheris, G.P. Morle, and J.M. Leiden. 1989. The first intron of the 4F2 heavy-chain gene contains a transcriptional enhancer element that binds multiple nuclear proteins. Mol. Cell. Biol. 9:25882597.

34. Chang, M.W., E. Barr, M.M. Lu, K. Barton, and J.M. Leiden. 1995. Adenovirus-mediated overexpression of the cyclin/CDK inhibitor, p21 inhibits vascular smooth muscle cell proliferation and neointima formation in the rat carotid artery model of ballon angioplasty. J. Clin. Invest. 96:2260-2268.

35. Barr, E., J. Carroll, A.M. Kalynych, S.K. Tripathy, K. Kozarsky, J.M. Wilson, and J.M. Leiden. 1994. Efficient catheter-mediated gene transfer into the heart using replication-defective adenovirus. Gene Ther. 1:51-58.

36. Lin, H., M.S. Parmacek, G. Morle, S. Bolling, and J.M. Leiden. 1990. Expression of recombinant genes in myocardium in vivo after direct injection of DNA. Circulation. 82:2217-2221.

37. Parmacek, M.S., and J.M. Leiden. 1989. Structure and expression of the murine slow/cardiac troponin C gene. J. Biol. Chem. 264:13217-13225.

38. Li, L., J.M. Miano, B. Mercer, and E.N. Olson. 1996. Expression of the SM22 $\alpha$ promoter in transgenic mice provides evidence for distinct transcriptional regulatory programs in vascular and visceral smooth muscle cells. J. Cell Biol. 132:849-859.

39. Moessler, H., M. Mericskay, Z. Li, S. Nagl, D. Paulin, and J.V. Small. 1996. The SM22 promoter directs tissue-specific expression in arterial but not in venous or visceral smooth muscle cells in transgenic mice. Development. 122: $2415-2425$.

40. Nabel, E.G., Z. Yang, S. Liptay, H. San, D. Gordon, C.C. Haudenschild, and G.J. Nabel. 1993. Recombinant platelet-derived growth factor B gene expression in porcine arteries induce intimal hyperplasia in vivo. J. Clin. Invest. 91:1822-1829

41. Nabel, E.G., L. Shum, V.J. Pompili, Z.Y. Yang, H. San, H.B. Shu, S Liptay, L. Gold, D. Gordon, and R. Derynck. 1993. Direct transfer of transforming growth factor beta 1 gene into arteries stimulates fibrocellular hyperplasia. Proc. Natl. Acad. Sci. USA. 90:10759-10763.

42. Nabel, E.G., Z.Y. Yang, G. Plautz, R. Forough, X. Zhan, C.C. Haudenschild, T. Maciag, and G.J. Nabel. 1993. Recombinant fibroblast growth factor-1 promotes intimal hyperplasia and angiogenesis in arteries in vivo. $\mathrm{Na}$ ture (Lond.). 362:844-846.

43. Pompili, V.J., D. Gordon, H. San, Z. Yang, D.W. Muller, G.J. Nabel, and E.G. Nabel. 1995. Expression and function of a recombinant PDGF B gene in porcine arteries. Arterioscler. Thromb. Vasc. Biol. 15:2254-2264.

44. Arbuthnot, P.B., M.P. Bralet, C. Le Jossic, J.F. Dedieu, M. Perricaudet, C. Brechot, and N. Ferry. 1996. In vitro and in vivo hepatoma cell-specific expression of a gene transferred with an adenoviral vector. Hum. Gene Ther. 7: 1503-1514. 